\title{
Integrated sensor analysis for GRACE - development and validation
}

\author{
B. Frommknecht ${ }^{1}$, H. Oberndorfer ${ }^{1}$, F. Flechtner ${ }^{2}$, and R. Schmidt ${ }^{2}$ \\ ${ }^{1}$ Institute for Astronomical and Physical Geodesy, Technical University of Munich, Arcisstrasse 21 D-80333, Munich, \\ Germany \\ ${ }^{2}$ GeoForschungsZentrum Potsdam, Germany
}

\begin{abstract}
The focus of this article is on the accelerometer on board the two GRACE (Gravity Recovery and Climate Experiment) satellites. In a first analysis the accelerometer system is studied. The behavior of the test mass and its capacitive feedback system is simulated in the time and the frequency domain for one degree of freedom. Only linear accelerations are considered so far. The second part of the analysis is about the practical implementation of the simulation model: Non-gravitational forces were derived from the GFZ (GeoForschungsZentrum) EPOS (Earth Parameter and Orbit System) software. In a closed loop the accelerometer measurements derived from these data in connection with simulated tracking data have been used for orbit recovery including the estimation of biases and scale factors for the accelerometer data. Details and results of this procedure are presented.
\end{abstract}

Key words. GRACE - sensor analysis - accelerometer

\section{Introduction}

GRACE succeeds the CHAMP (Challenging Minisatellite Payload) mission in the area of Earth gravity field measurements. The anticipated increase in accuracy will be achieved by utilizing the measurement of the relative motion between the two satellites (labelled as GRACE-A and GRACE-B) following each other on the same orbital track. The two satellites are interconnected by a K-band microwave link to measure the exact separation and its rate of change to an accuracy of about $1 \mu \mathrm{m} / \mathrm{s}$. In order to take into account the nongravitational forces, their size as well as their orientation in space, both satellites will be equipped with star cameras and accelerometers. The position and velocity of the satellites will be measured using onboard GPS receivers.

The purpose of the integrated sensor analysis is to provide a simulator of the gravity measurement system of the

Correspondence to: B. Frommknecht

(frommknecht@bv.tum.de)
GRACE mission. It is based on mathematical models of the individual sensors (accelerometer, star sensors, etc.) as well as of their interaction. The focus of this paper is on the accelerometer part of GRACE mission and it is divided into two parts. In the first part, the behavior of the accelerometer in the time and frequency domain is studied. Then, in part two, we deal with its practical implementation: Non-gravitational forces were simulated using the GFZ EPOS software. In a closed loop the simulated accelerometer measurements derived from these data in connection with simulated tracking data have been used for orbit recovery including the estimation of biases and scale factors for the accelerometer data. Details and results of this procedure are presented.

\section{Development of the accelerometer model}

\subsection{Introduction}

The accelerometer on board of the GRACE satellites (see Fig. 1) is a three axis capacitive accelerometer with two sensitive and one less sensitive axes. The sensitive axes point into flight and into radial direction, the less sensitive axis points into cross-track direction (coplanar to orbit plane, normal to flight direction). According to specification the accuracy of the sensitive axes is $10^{-10}\left[\mathrm{~m} / \mathrm{s}^{2}\right]$, and that of the less sensitive axis $10^{-9}\left[\mathrm{~m} / \mathrm{s}^{2}\right]$. The proof mass is a $40 \mathrm{x}$ $40 \times 10 \mathrm{~mm}$ gold coated cube made of titan, weighing $72 \mathrm{~g}$. The proof mass is connected to the accelerometer frame with a gold wire that enables to control the charge of the proof mass. There are twelve electrodes forming six pairs that allow control of proof mass motion.

The purpose of the accelerometer is the measurement of the non-gravitational forces acting on the GRACE-satellites. The accelerometer system consists of two parts: the position detection mechanism, that measures the position of the proof mass and the servomechanism, that drives the proof mass back to its nominal position. In the following sections, these constituents of the accelerometer are described for one 


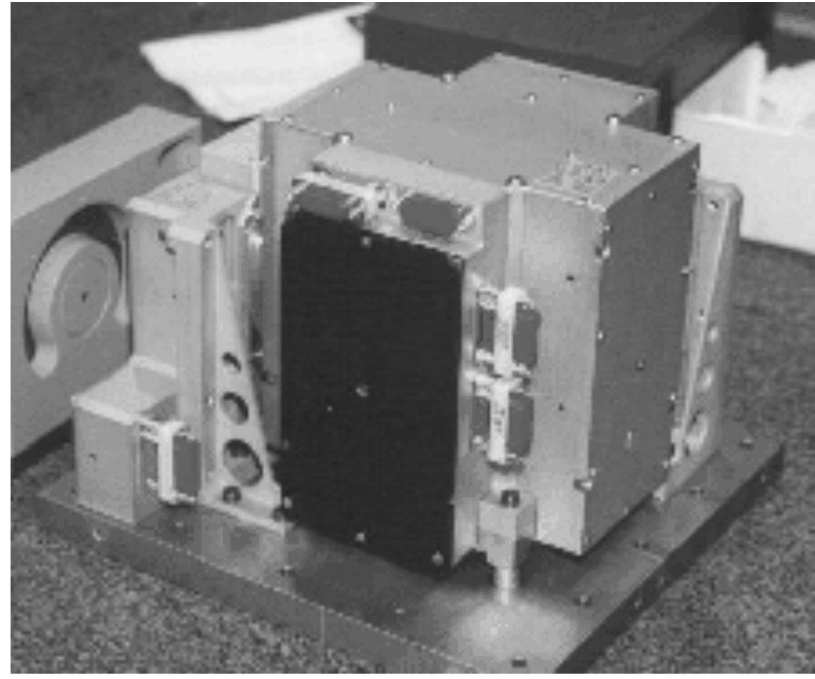

Fig. 1. SuperSTAR accelerometer on board the GRACE satellites, produced by ONERA, France.

degree of freedom, the principle of operation is analogous for the two other axes.

\subsection{Logical model}

Figure 2 shows the schematic design for one axis. The proof mass is between two electrodes, charged with voltage $+V$ and $-V$, respectively. The proof mass is charged with a voltage $V_{t}$ consisting of the polarization voltage $V_{p}$ and an alternating current, the detection voltage $V_{d}$ :

$V_{t}=V_{p}+V_{d}(t)$.

The frequency of the detection voltage is about $100 \mathrm{kHz}$, too high to affect the motion of the proof mass. The nominal position of the proof mass is in the middle between the electrodes, with no off-set $x=0$. Between the walls of the proof mass and the electrodes two electric fields $E_{1}$ and $E_{2}$ are forming. If $V$ and $V_{t}$ are considered positive and constant, one recognizes that the proof mass will start to move towards the electrode charged with $-V$. Through this movement the gap between proof mass and electrode reduces, increasing the field and the attraction. In this configuration the accelerometer system is inherently unstable and servo control of the proof mass motion is mandatory. A capacitive detector measures the position of the proof mass by comparing the capacitances. Then a feedback loop including a PID (Proportional Integrative Derivative) controller determines the control voltage $V$ to keep the proof mass motionless in its nominal position.

\subsection{Mathematical model}

In this section the equation of motion of the proof mass is derived. First, we have to find a linear expression for the capacitive force acting on the proof mass. This expression can then be linked to the non-gravitational forces acting on

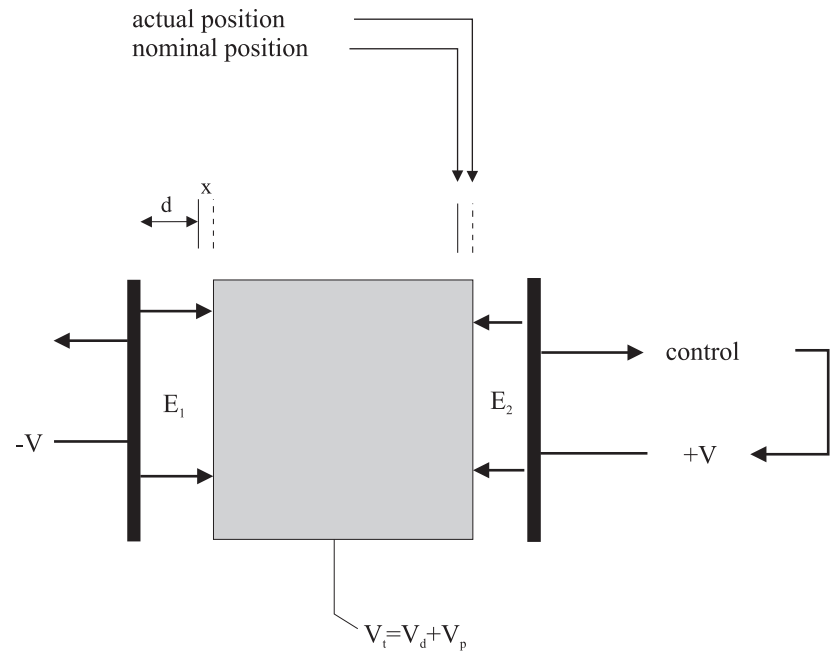

Fig. 2. Concept of a capacitive accelerometer for one axis.

the satellite. For the capacitive force, after Coloumb's law we have:

$F_{c a p, i}=\frac{1}{2} \epsilon_{0} A E_{i}^{2}$ for $i=1,2$

where

$\begin{array}{ll}\epsilon_{0} & \text { the dielectric constant, } \\ A & \text { electrode area }\left[\mathrm{m}^{2}\right], \\ E & \text { electric field force }[\mathrm{J}] .\end{array}$

As the attraction of field $E_{1}$ is opposite to the attraction of field $E_{2}$, we derive for the overall capacitive force:

$F_{\text {cap }}=\frac{1}{2} \epsilon_{0} A\left(E_{1}^{2}-E_{2}^{2}\right)$.

Considering $E=\frac{U}{s}$, where $U$ is the voltage between proof mass and electrode and $s=d+x$ is the distance between proof mass and electrode, and averaging $V_{d}(t)$ over time we get (according to Josselin et al., 1999; Touboul et al., 1999):

$$
\begin{aligned}
F_{c a p}= & \frac{1}{2} \epsilon_{0} A\left(\frac{\left(V^{2}-2 V V_{p}+\bar{V}_{t}^{2}\right)}{(d-x)^{2}}\right. \\
& \left.-\frac{\left(V^{2}+2 V V_{p}+\bar{V}_{t}^{2}\right)}{(d+x)^{2}}\right)
\end{aligned}
$$

with

$$
\begin{aligned}
\bar{V}_{t}^{2} & =V_{p}^{2}+\bar{V}_{d}^{2}, \\
\bar{V}_{d} & =\frac{\max \left(V_{d}(t)\right)}{\sqrt{2}} .
\end{aligned}
$$

This expression is nonlinear both in the displacement $x$ of the proof mass and in the control voltage $V$, but becomes linear in the voltage $V$ for $x=0$. Expansion into a Taylor series for small $x / d$ and neglecting higher order terms yields:

$$
F_{c a p}(V, x)=-\frac{2 \epsilon_{0} A}{d^{2}}\left[V V_{p}-\frac{x}{d} \bar{V}_{t}^{2}\right] \text {. }
$$


As mentioned before, the control voltage $V$ is not constant, it is controlled by a PID controller. After Smit (2000) it is:

$V(x)=K_{P I D} \omega_{0}^{2}\left(\dot{x} / \omega_{d}+x+\omega_{i} \int^{t} x \mathrm{~d} t\right)$

with

$$
\begin{array}{ll}
K_{P I D} & \text { overall gain, } \\
\omega_{0}^{2} & \text { natural frequency of PID-controller, } \\
\omega_{d} & \text { characteristic frequency for derivative part, } \\
\omega_{i} & \text { characteristic frequency for integral part. }
\end{array}
$$

The capacitive system is used as an accelerometer. It is exposed to an external force $F_{\text {ext }}$, resulting from the non-conservative forces acting on the satellite. The control voltage $V$ is adjusted so that the proof mass stays in its nominal position. The equation of motion of the proof mass can now be stated as:

$$
M \ddot{x}=F_{\text {ext }}+F_{\text {cap }}(V(x), x) .
$$

Insertion of the linearized expressions from Eqs. (7) and (8) yields:

$$
\begin{aligned}
\ddot{x}= & -\frac{2 \epsilon_{0} A V_{p} K_{P I D}}{M d^{2}} \omega_{0}^{2}\left(\dot{x} / \omega_{d}+x+\omega_{i} \int x \mathrm{~d} t\right) \\
& +\frac{2 \epsilon_{0} A \bar{V}_{t}^{2}}{M d^{3}} x+F_{\text {ext }} / M .
\end{aligned}
$$

This expression is in agreement with the one given by Stanton et al. (1998).

\subsection{Measurement model}

The measurement model is the connection between the accelerations due to non-conservative forces acting on the satellite and the output of the accelerometer. In a first step the accelerations that act on the proof mass are derived, in a second step the connection to the measurement (output) of the accelerometer is established.

If there is a displacement of the accelerometer relative to the center of mass of the satellite, not only accelerations due to the nonconservative forces but also accelerations due to gravitational forces and angular motion act on the proof mass:

$\bar{\gamma}_{A}=\left(\underline{G}_{i j}+\underline{\Omega}_{j k} \underline{\Omega}_{i k}+\underline{\dot{\Omega}}_{i k}\right) \delta \overline{\mathrm{r}}+\bar{\gamma}_{D r a g}$

with

$\bar{\gamma}_{A} \quad$ acceleration on proof mass,

$\underline{G}_{i j} \quad$ matrix of gravity gradients,

$\underline{\Omega}_{i k} \quad$ angular velocity of satellite,

$\underline{\dot{\Omega}}_{i k} \quad$ angular acceleration of satellite,

$\delta \overline{\mathrm{r}} \quad$ displacement of accelerometer

from center of mass.

$\bar{\gamma}_{\text {Drag }} \quad$ acceleration due to nonconservative forces (drag)

In this study, the displacement from the center of mass has

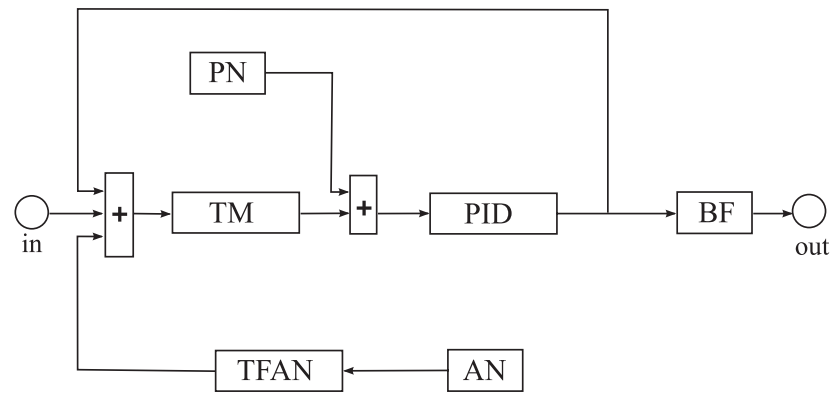

Fig. 3. Dynamic measurement model for one axis.

been neglected, thus the acceleration on the proof mass becomes:

$\bar{\gamma}_{a}=\bar{\gamma}_{\text {Drag }}$.

Next the connection between external accelerations and the measurement of the accelerometer is investigated. Oberndorfer (2000) states as measurement model:

$\bar{\Gamma}_{a}=\underline{K}_{0, A}+\underline{K}_{1, A} \bar{\gamma}_{A}+\underline{K}_{l m, A} \bar{\gamma}_{A}+\underline{K}_{2, A} \bar{\gamma}_{A} \bar{\gamma}_{A}+$ noise.(13)

with

$\bar{\Gamma}_{a} \quad$ measured acceleration,

$\underline{K}_{0, A} \quad$ bias,

$\underline{K}_{1, A} \quad$ scale factor (linear),

$\underline{K}_{2, A} \quad$ quadratic factor,

In this

$\underline{K}_{l m, A}$ coupling and misorientation matrix,

noise measurement noise.

study, we neglected the quadratic factor and the coupling and misorientation of the accelerometer axes. The noise consists of two terms: acceleration noise and position noise. Acceleration noise is white with an additional $1 / f$ component below a turnover frequency $f_{0}$ :

noise $_{a}(f)=A_{a} \sqrt{1+f_{0} / f} \quad\left[\mathrm{~ms}^{-2} / \sqrt{\mathrm{Hz}}\right]$.

Position noise is white:

noise $_{p}(f)=A_{p} \quad\left[\mathrm{~ms}^{-2} / \sqrt{\mathrm{Hz}}\right]$.

Thus, we arrive at:

$\bar{\Gamma}_{a}=\underline{K}_{0, A}+\underline{K}_{1, A} \bar{\gamma}_{A}+$ noise $_{a}(f)+$ noise $_{p}(f)$.

\subsection{Dynamic measurement model}

The above mentioned measurement model does not consider the dynamics of the measurement process. To consider the dynamics we have implemented Eq. (10) in SIMULINK, a MATLAB toolbox used for the simulations. Figure 3 shows the dynamic model. External accelerations enter to the left and are then passed to the block denoted as 'TM', that represents the proof mass dynamics, i.e. the transfer from accelerations on the proof mass to a new position of the proof mass. The position detection mechanism is simulated by adding position noise ('PN') to the proof mass position. This position 


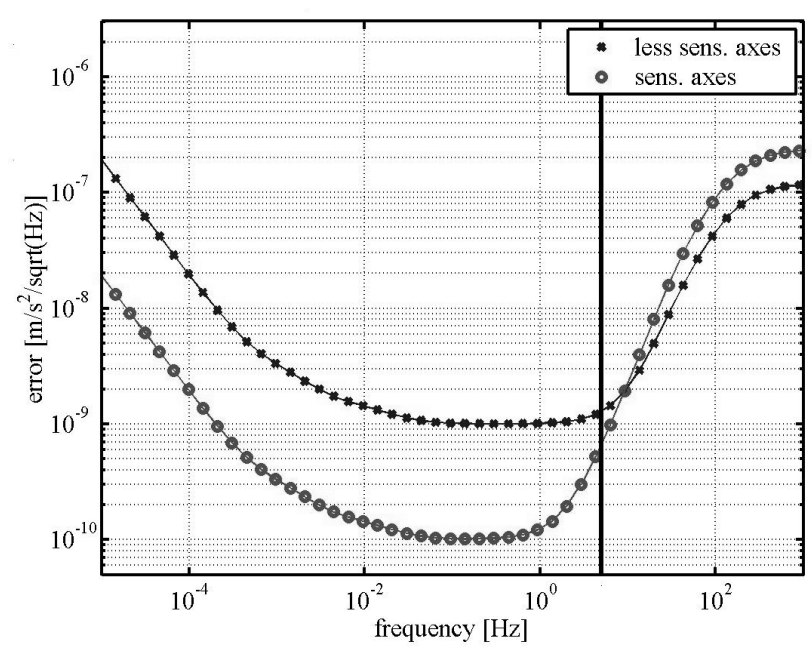

Fig. 4. Root PSD (Power Spectral Density) of the dynamic acceleration measurement error due to noise. Upper line represents less sensitive axis, lower line sensitive axes. The vertical black line is the Nyquist frequency of accelerometer output sampling rate $(5 \mathrm{~Hz})$. The error rises towards the higher frequencies due to position noise and towards the lower frequencies due to acceleration noise.

is then passed to the block denoted as 'PID', that implements the PID-controller. Via feedback the controller exerts a corrective acceleration on the proof mass to move it back to its nominal position. The acceleration noise is modelled as an additional acceleration in the block 'AN'. At the output of the accelerometer a third order butterworth filter ('BF') is applied for the purpose of anti-aliasing.

Let us now have a look at the dynamic error due to noise (see Fig. 4). The rise towards lower frequencies is because of the acceleration noise. The acceleration noise of the sensitive axes is lower than that of the less sensitive axis. Acceleration noise combines all effects that induce parasitic accelerations on the proof mass: stiffness of the gold wire, Lorentz force, influence of the earth's magnetic field and others. The rise towards higher frequencies is due to the position noise. We notice that position noise of the sensitive axes is higher than that of the less sensitive axis. The black line indicates the Nyquist frequency of the designated output frequency of the accelerometer $(10 \mathrm{~Hz})$. We note that there is much energy in the error signal at frequencies of $5 \mathrm{~Hz}$ and above.

If the signal would be sampled unchanged, aliasing would occur and spoil the measurement. That is the reason for the implementation of an anti-aliasing filter at the output of the accelerometer, a third order butterworth filter with a cut-off frequency of $3 \mathrm{~Hz}$. The effect of this filter is depicted in Fig. 5. The anti-aliasing filter effectively reduces the signal energy at higher frequencies, so that no degradation of the measurement will occur.

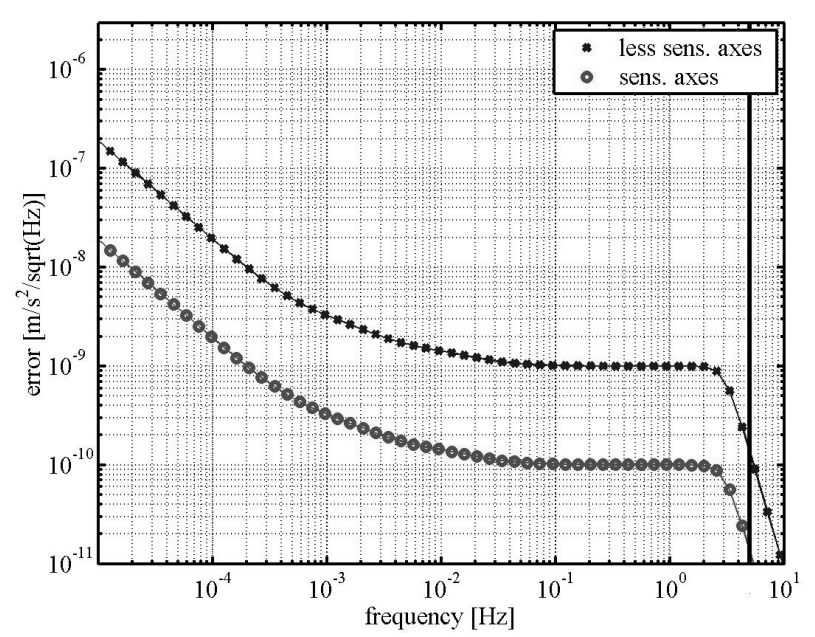

Fig. 5. Root PSD of the dynamic measurement error at the output of the accelerometer. Upper line represents less sensitive axis, lower line sensitive axes. The vertical black line is Nyquist frequency of accelerometer output sampling rate $(5 \mathrm{~Hz})$. In contrast to Fig. 4 the signal energy at frequencies of $5 \mathrm{~Hz}$ and above is diminished through the application of the butterworth filter.

\section{Simulation}

\subsection{Introduction}

In order to validate the above described sensor analysis tools a numerical simulation close to the planned real-world GRACE scenario was conducted. The simulation consisted of three steps: First, GRACE orbits based on realistic initial values were integrated numerically, using GFZ's EPOS software. This step provides ephemeris and time series of the non-conservatives forces along the orbit of the two spacecrafts. In this study the non-conservatives forces the dominating effects resulting from atmospheric drag and solar radiation pressure were considered. For the evaluation of the modelled non-conservative forces a realistic surface model of the GRACE satellites has been applied. Additionally, GPS (Global Positioning System) high-low satellite-tosatellite measurements (pseudo-range $\&$ phase) were generated during this step to be used later in orbit recovery. In the next step, simulated accelerometer measurements including bias, scale factors and noise were generated from the time series of the non-conservative forces using the TUM software. Finally, a dynamic orbit recovery (using EPOS) based on this simulated accelerometer data and the GPS tracking data was carried out. The following list summarizes the simulation environment of step 1:

- Gravitational accelerations (gravity field model EIGEN$1 \mathrm{~S}$ up to degree 100)

- Tidal accelerations from third bodies (sun and moon). Both, the sun and the moon were assumed to be point masses. 

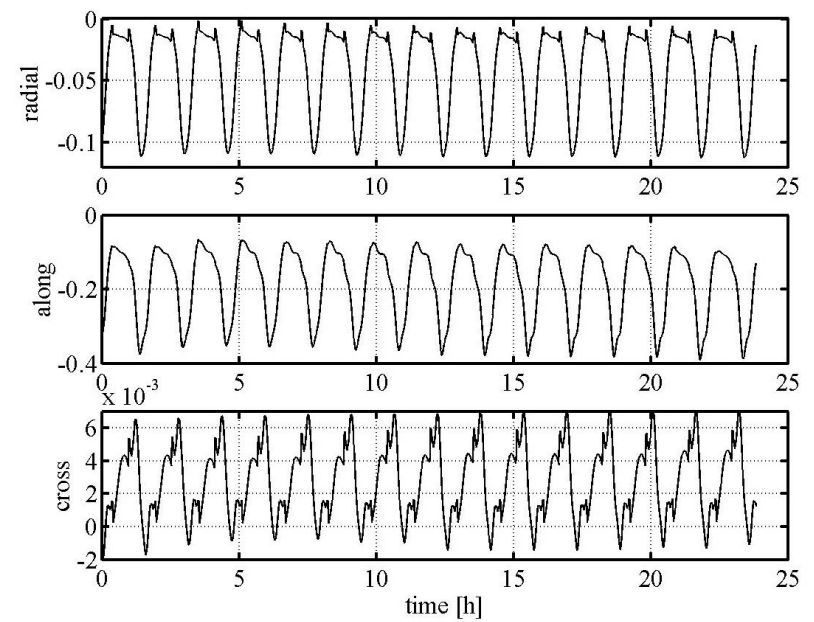

Fig. 6. Time series of non-conservative forces (atmospheric drag plus solar radiation) along the orbit of GRACE-A. Accelerations given in $\left[\frac{\mu m}{s^{2}}\right]$.

- Atmospheric drag was simulated using CIRA86.

- Solar radiation pressure was considered.

- Macro model of the GRACE spacecrafts (identical for each satellite) applied.

- The step size of the numerical integration procedure, time series of the non-conservative forces and the simulated GPS observational data was 5 seconds.

- Arc length was 1 day.

Figure 6 depicts the time series of the sum of atmospheric drag and solar radiation pressure derived from above listed models along the orbit of satellite GRACE-A in the conventional orbital system (radial axis collinear with position vector $\boldsymbol{r}(t)$, cross-track perpendicular to radial axis as $\boldsymbol{e}_{\text {cross }}=$ $\boldsymbol{r}(t) \times \dot{\boldsymbol{r}}(t)$, along-track completes the right-hand triad; $\boldsymbol{r}(t)$ and $\dot{\boldsymbol{r}}(t)$ are the position vector and the velocity vector of the spacecraft at epoch in inertial frame). The signals show characteristic patterns of low orbiting satellites such as prevailing once-per-revolution oscillations, entry and exit into/from Earth's shadow (peaks in radial direction), and so on. Typically, the most dominant effect is the acceleration (respectively deceleration) of the spacecraft in along-track direction followed by that in radial direction. The sign of these two signals is negative thus causing the well-known decay of the orbit due to non-conservative forces. In cross-track direction, i.e. perpendicular to the orbital plane accelerations due to atmospheric drag and solar radiation are much smaller.

\subsection{Generation of simulated accelerometer measurements}

In order to generate simulated accelerometer measurements, Eq. (13) was applied to the simulated non-gravitational accelerations of Fig. 6. Table 1 shows the applied values for bias,
Table 1. Applied values for biases, scale factors and noise on all three axes of the accelerometer. Sensitive axes are radial and alongtrack, less sensitive axis is cross-track

\begin{tabular}{cccc}
\hline & $\begin{array}{c}\text { Bias } \\
{\left[\mathrm{m} / \mathrm{s}^{2}\right]}\end{array}$ & $\begin{array}{c}\text { Scale } \\
{[-]}\end{array}$ & $\begin{array}{c}\text { Noise } \\
{\left[\mathrm{m} / \mathrm{s}^{2}\right]}\end{array}$ \\
\hline Cross-track & $5 * 10^{-5}$ & 1.02 & $\begin{array}{c}\text { Pos: } 3 * 10^{-13} \\
\text { Acc: } 1 * 10^{-09}\end{array}$ \\
\hline Radial & $-2 * 10^{-6}$ & 0.98 & $\begin{array}{l}\text { Pos: } 6 * 10^{-13} \\
\text { Acc: } 1 * 10^{-10}\end{array}$ \\
\hline Along-track & $2 * 10^{-6}$ & 1.01 & Pos: $6 * 10^{-13}$ \\
& & & Acc: $1 * 10^{-10}$ \\
\hline
\end{tabular}

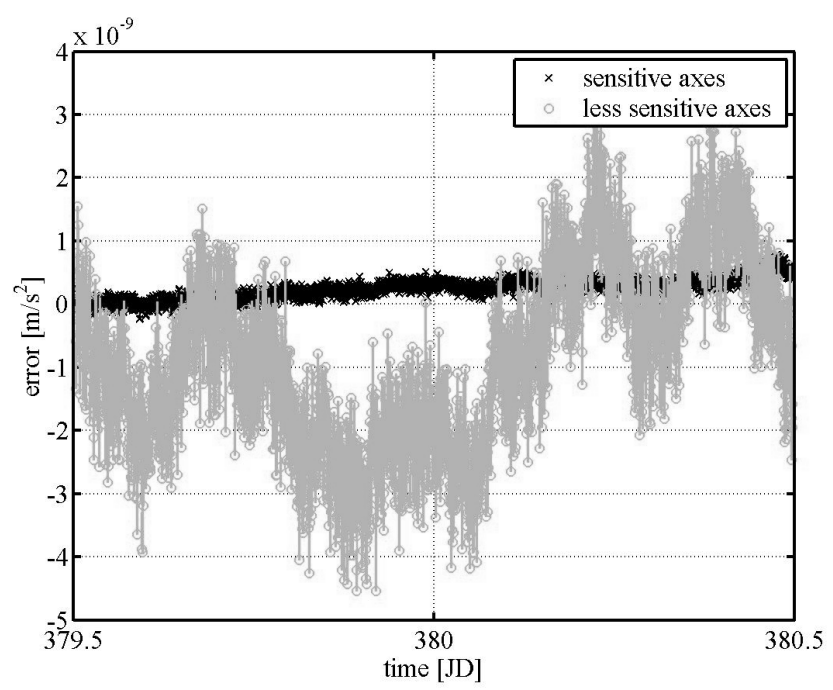

Fig. 7. Time series of simulated accelerometer measurement error due to noise.

scale factors and noise terms. Figure 7 shows the time series of the simulated accelerometer measurement error. As to be expected, the noise of the less sensitive axis is higher than the noise of the sensitive axes. A long term drift effect is present for both the sensitive and the less sensitive axes, but is more pronounced for the less sensitive component. The displayed time series of the simulated accelerometer measurement error is only one possible realization of the acceleration and the position noise. The behavior of the realization depends on the initial value for the noise processes. We have chosen a rather pessimistic realization to get conservative results.

These observations are confirmed by comparison with the theoretical error model (see Fig. 8).

The effect of the acceleration noise causes the PSD to rise towards the lower frequencies for both the sensitive and less sensitive axes. Again the noise level of the less sensitive axis is higher than the noise level of the sensitive axes. The solid black lines indicate the modelled measurement error, which is in good agreement with the simulation. 


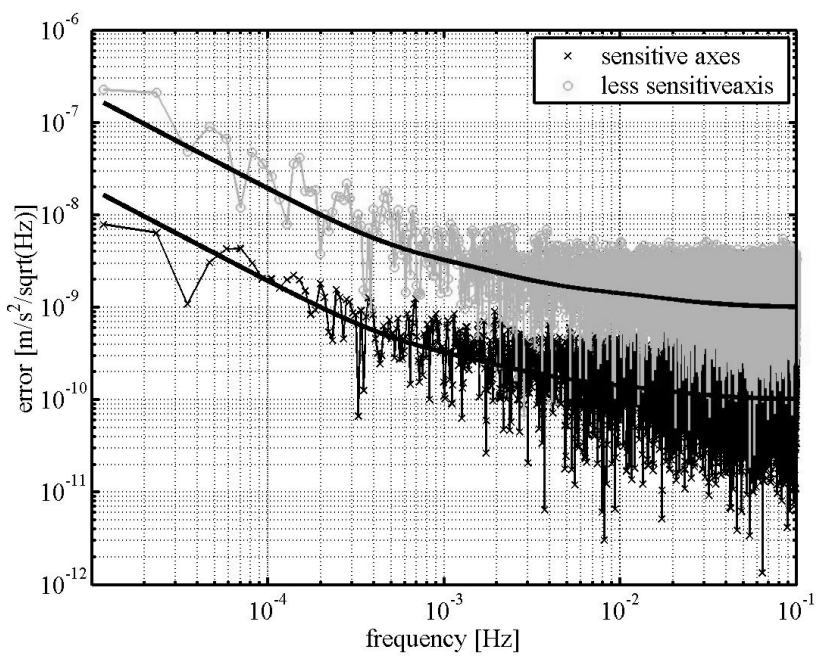

Fig. 8. Root PSD of the measurement error due to noise. Solid, thick black line represents error model.

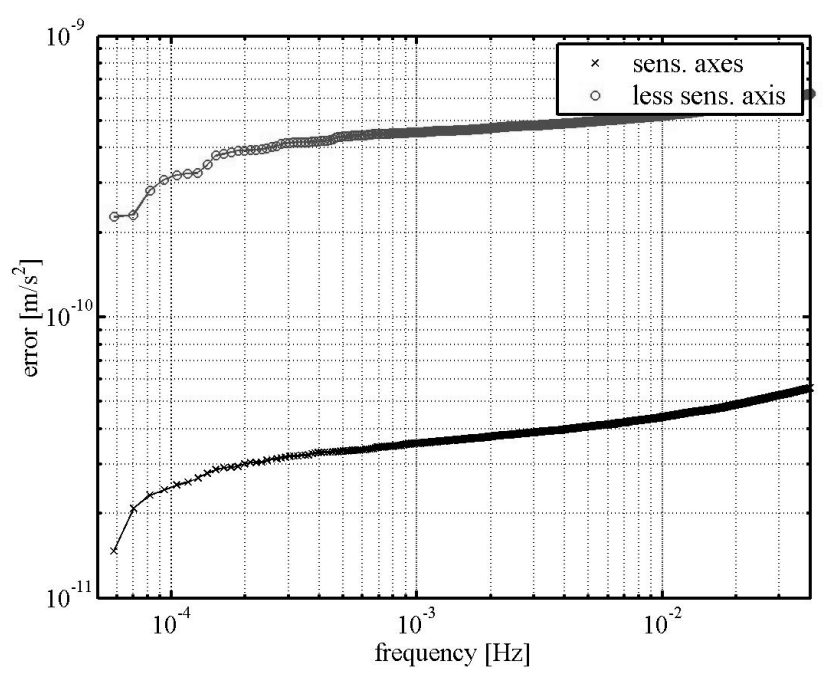

Fig. 9. Cumulative measurement error due to noise. Cumulative error is attained through integration over a certain frequency band.

To get a more detailed view of the measurement error Fig. 9 displays the cumulative error over the measurement bandwidth from $5 \times 10^{-5} \mathrm{~Hz}$ to $0.1 \mathrm{~Hz}$. It is noticed that the error stays below the measurement error specifications of $1 \times 10^{-9} \mathrm{~m} / \mathrm{s}^{2}$ and $1 \times 10^{-10} \mathrm{~m} / \mathrm{s}^{2}$ for the less sensitive axis and the sensitive axes, respectively, as some important error sources such as the coupling between the accelerometer axes and the center of mass offset have been neglected.

\subsection{Dynamic orbit recovery and estimation of accelerome- ter parameters}

For orbit recovery a dynamic method in combination with a least-squares adjustment procedure implemented in EPOS has been used. The force modelling of conservative forces was identical to the one used in the simulation (see Sect. 3.1).
Table 2. Estimated accelerometer parameters during the dynamic orbit recovery for satellite GRACE-A. In bold letters are the values applied for the generation of the simulated accelerometer data

\begin{tabular}{|c|c|c|}
\hline GRACE-A & $\begin{array}{c}\text { Bias } \\
{\left[\mathrm{m} / \mathrm{s}^{2}\right]}\end{array}$ & $\begin{array}{c}\text { Scale } \\
{[-]}\end{array}$ \\
\hline Cross-Track & $\begin{array}{c}4.880 * 10^{-5} \pm 0.5 * 10^{-7} \\
\mathbf{5} * \mathbf{1 0}^{-\mathbf{5}}\end{array}$ & $\begin{array}{c}1.0245 \pm 0.9 * 10^{-3} \\
\mathbf{1 . 0 2}\end{array}$ \\
\hline Radial & $\begin{array}{c}-2.042 * 10^{-6} \pm 0.6 * 10^{-9} \\
-\mathbf{2} * \mathbf{1 0}^{-\mathbf{6}}\end{array}$ & $\begin{array}{c}0.9765 \pm 0.2 * 10^{-3} \\
\mathbf{0 . 9 8}\end{array}$ \\
\hline Along-Track & $\begin{array}{c}1.977 * 10^{-6} \pm 0.2 * 10^{-10} \\
\mathbf{2} * \mathbf{1 0}^{-\mathbf{6}}\end{array}$ & $\begin{array}{c}1.0116 \pm 0.6 * 10^{-5} \\
\mathbf{1 . 0 1}\end{array}$ \\
\hline
\end{tabular}

The models for the non-conservative forces (i.e. atmospheric drag and solar radiation pressure) were replaced by the biased, scaled and noisy simulated accelerometer measurements. Since the simulated accelerometer data is introduced as true acceleration acting on the individual satellite, in particular due to the applied accelerometer noise the simulated system becomes inconsistent. However, during the adjustment process the discrepancies will be mapped into the estimated parameters and the effect of bias, scale and accelerometer noise can be studied. To be estimated are initial elements of GRACE-A and GRACE-B, accelerometer parameters for bias and scaling for each component of the axes of each accelerometer and ambiguities for the GPS pseudo-ranges. Table 2 shows the results for the estimated accelerometer parameters for GRACE-A. (Results for GRACE-B are equivalent.) In bold the reference values from Table 1 are given. As can been seen all estimated parameters are in principle in good agreement with the applied reference value. Deviations for scale factors are below 1 percent. The biases show larger discrepancies at the level of few percents. In addition, the formal errors $\sigma_{i}$ in relation to the absolute value of the solved-for parameter, indicate less accurate results for the bias parameters. This difference between scale and bias estimation seems to originate from the fact that the applied accelerometer noise introduces some long term drift in the accelerometer data (compare Fig. 8) which is - at least for some constant part - mapped into the estimated bias parameters. The estimation of scaling factors is seemingly less affected by accelerometer noise than for the bias parameters. When reading Table 2 row-wise one can see differences for the individual axis. As expected the less sensitive axis - cross-track - shows largest deviations with respect to the absolute values (bias and scale), but also less parameter accuracy. This is due to the larger values for the applied bias and accelerometer noise, but also due to the rather small magnitude of accelerations acting on the spacecraft in this direction. The smallest deviations from the reference values and the best formal accuracy is observable for parameters in along-track direction. In radial direction - where the sensitivity of the accelerometer should be equivalent to the along-track component - the obtained results are worse than for the along-track axis. This 
discrepancy can be explained by the different signal strength of the non-conservative forces, prevailing in the along-track direction.

\section{Conclusion and outlook}

Integrated sensor analysis tools for three axis capacitive accelerometers have been developed. In this study, time series of simulated accelerometer data considering biases, scale factors and noise were derived from time series of simulated non-conservative forces for one day of simulated GRACE data. Analysis of generated accelerometer data in the time and frequency domain shows consistent performance of the software. In a closed-loop simulation this spurious accelerometer data has been applied into dynamic orbit recovery. Overall results show that the bias and scale parameters are reliably estimated. Varying results for estimated bias and scale parameters indicate that part of the low frequency contribution of the accelerometer noise is mapped into bias parameters. The determination of scale factors is less affected by accelerometer noise, however. Due to the different sensitivity of the accelerometer axis and the different magnitude of the accelerations the quality of the estimation of bias and scale parameters in the radial, cross- and along-track direction differs. In this study the most reliable results were obtained for the along-track parameters, followed by the radial and cross-track components. At this stage, with a reliable tool for the simulation of realistic accelerometer data at hand, detailed numerical investigations, e.g. of the influence of ac- celerometer errors on gravity field recovery of the GRACE mission become feasible. Additional models for further accelerometer errors such as coupling or center-of-mass offsets are implemented in the simulation software. The resulting effects on orbit determination and gravity recovery will be studied on a numerical basis and compared to results from real world data.

Acknowledgements. This study was carried out under the grant of the German Federal Ministry of Education and Research (BMBF) No. 03F0326A. We also wish to thank the referee for his valuable comments.

\section{References}

Josselin, V., Touboul, P., and Kielbasa, R.: Capacitive detection scheme for space accelerometers applications, Sensors and Actuators, Elsevier Science Ltd., 78, 92-98, 1999.

Oberndorfer, H.: Integrierte Sensoranalyse am Beisiel des Satelliten CHAMP, Tech. Rep. Mu 1141/2-1, Institut für Physikalische und Astronomische Geodäsie, TU München, 2000.

Smit, J. M.: GOCE End to End Closed Loop Simulation, workpackage 210, GOCE End to End Performance Analysis, ESTEC Contract No.12735/98/NL/GD, 1, 9-30, 2000.

Stanton, R., Bettadpur, S., Dunn, C., Renner, K.-P., and Watkins, M.: GRACE Science \& Mission Requirements Document, Tech. Rep. 327-200, Jet Propulsion Laboratory, 1998.

Touboul, P., Foulon, B., and Willemont, E.: Electrostatic Space Accelerometers for Presend And Future Missions, Acta Astronautica, Elsevier Science Ltd., 45, 605-617, 1999. 